\title{
HERBRAND E A SILOGÍSTICA AMPLIADA $^{1}$
}

Frank Thomas Sautter (UFSM) ${ }^{2}$

ftsautter@ufsm.br

Resumo: Forneço um método de prova para a Lógica de Predicados Monádicos de Primeira Ordem. Esse método utiliza a Forma Normal de Herbrand e as Formas Normais Disjuntiva e Conjuntiva da Lógica Proposicional. A validade é determinada por mera inspeção da presença e disposição de fórmulas que atuam como átomos de informação. Mediante a Forma Normal de Herbrand se estabelece a relação exata entre a Lógica de Predicados Monádicos de Primeira Ordem e a silogística ampliada desenvolvida ao longo do século XIX.

Palavras-chave: Conhecimento simbólico; Lógica de Predicados Monádicos; Silogística ampliada; Herbrand.

\section{MOTIVAC̣ÃO}

Os métodos diagramáticos de prova, ao contrário dos métodos simbólicos, costumam apresentar a característica da sinopticidade (inspecionabilidade global), ou seja, a capacidade para apresentar uma prova como um todo, em um coup d'oeil $l^{3}$. A característica da sinopticidade é a mais destacada característica dos métodos diagramáticos de prova, mas não é a única, e talvez nem mesmo a mais importante

\footnotetext{
${ }^{1}$ Recebido: 31-03-2015/Aprovado: 23-07-2015/Publicado on-line: 31-08-2015.

${ }^{2}$ Frank Thomas Sautter é Professor Associado no Departamento de Filosofia da Universidade Federal de Santa Maria, Santa Maria, RS, Brasil.

${ }^{3}$ Secco (2013) distingue entre inspecionabilidade global e inspecionabilidade local. Essa última é a capacidade para examinar uma prova passo a passo.
} 
em termos fundacionais.

Consideremos o seguinte diagrama de Venn:

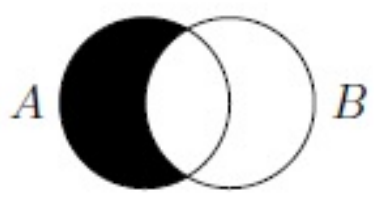

Figura 1: Diagrama de Venn para "Todo A é B".

Nesse diagrama está representada a informação de que não há objetos com o atributo $A$ e sem o atributo $B$; está representada a ausência de informação sobre a existência de objetos com os atributos $A$ e $B$; e está representada a ausência de informação sobre a existência de objetos com o atributo $B$ e sem o atributo $A^{4}$. Essa caracterização dos diagramas de Venn - elementos gráficos salientes que veiculam informação - também é frequente em outros métodos diagramáticos de prova. Ela é uma característica não apenas epistemologicamente relevante, como o é a sinopticidade, mas também é fundacionalmente relevante, pois, por seu intermédio, é possível antever um tratamento exclusivamente informacional das noções lógicas. Por exemplo, a noção de validade de um argumento pode ser definida do seguinte modo:

Definição 1 (Validade). Um argumento é válido se, e somente se, a informação veiculada pela conclusão é uma parte, própria ou imprópria, da fusão mereológica das informações veiculadas pelas premissas.

\footnotetext{
${ }^{4}$ Mendonça (2013) observa que a possibilidade de representar conhecimento parcial, ou seja, a possibilidade de representar, em um mesmo diagrama, a presença de informação e a ausência de informação, constitui a principal crítica de Venn aos diagramas de Euler.
} 
A noção de informação apresenta maior granularidade do que a noção de verdade, quer dizer, ao utilizar a noção de informação podemos fazer mais e melhores distinções do que aquelas que podemos fazer com a noção de verdade. Isso nos permite tratar, mediante a noção de informação, tanto das noções tradicionais da lógica como de noções lógicas que, embora relevantes, temos grande dificuldade para tratar por intermédio da noção de verdade. Por exemplo, noções de dinâmica da argumentação tal como aquelas relativas ao "conserto" de argumentos, seja pelo acréscimo de informação relevante às premissas de um argumento inválido, seja pela supressão de informação irrelevante das premissas de um argumento válido, são de difícil tratamento mediante a noção de verdade.

Quanto ao caráter diagramático do método aqui apresentado, Sautter (2013) previamente desenvolveu um método de prova para a Lógica Proposicional Clássica em que elementos salientes das representações veiculam informação e, por isso, permitem o teste de validade por mera inspeção visual, ou seja, mediante operações simples de constatação de determinados elementos salientes em determinadas disposições, sem que nenhuma operação mais complexa se faça necessária. Esse método tem ainda, como característica distintiva, a utilização de ideogramas: as fórmulas da Lógica Proposicional Clássica são, relativamente ao método, ideogramas, ou seja, elas são híbridos entre representações gráficas e não-gráficas. Por um lado, a função ectética ${ }^{5}$, típica de métodos diagramáticos de prova, não está presente; por outro lado, a função operativa ${ }^{6}$, típica dos métodos simbólicos

\footnotetext{
${ }^{5}$ A estrutura das representações reproduz a estrutura dos representados.

${ }^{6}$ Obtenção de conhecimento por cálculo com as representações.
} 
de prova, não está presente ${ }^{7}$. Essa utilização de ideogramas deriva de um trabalho anterior de Sautter (2012), que, por sua vez, deriva de uma prática de Peirce. A utilização de ideogramas permite a discussão in concreto do problema de demarcação entre representação gráfica e representação não-gráfica. ${ }^{8}$

O objetivo deste trabalho é desenvolver um método de prova para a Lógica de Predicados Monádicos de Primeira Ordem ${ }^{9}$ com as mesmas características do método desenvolvido por Sautter (2013). Esse método de prova para a Lógica Proposicional Clássica utiliza o seguinte algoritmo:

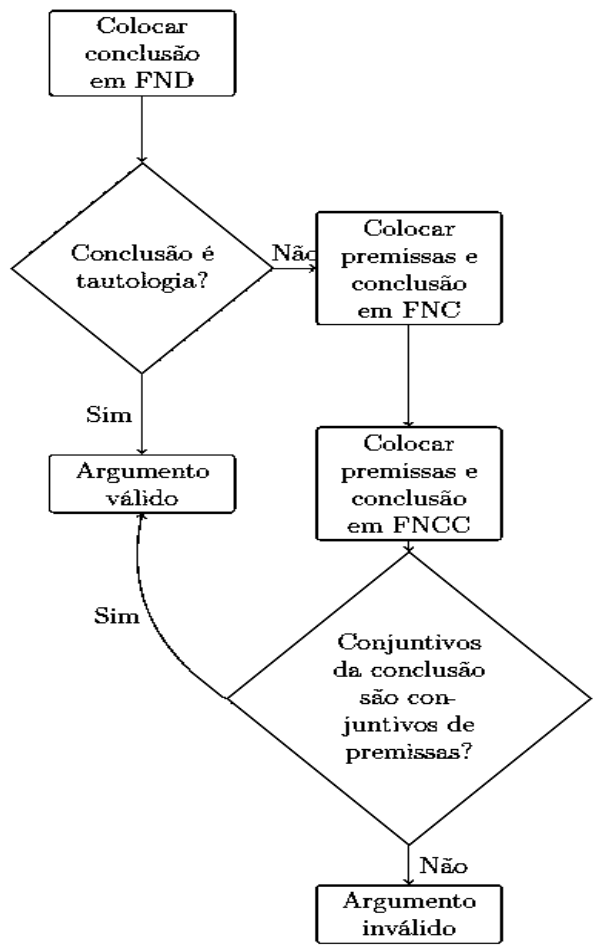

Figura 2: Método de prova para Lógica Proposicional Clássica: "FND" é a forma normal disjuntiva, "FNC" é a forma normal conjuntiva, e "FNCC" é a forma normal conjuntiva completa relativa às proposições atômicas do argumento.

\footnotetext{
${ }^{7}$ Utilizo a terminologia de Esquisabel (2012), que apresenta as várias funções do conhecimento simbólico sob uma perspectiva leibniziana.

${ }^{8}$ Shimojima (2001) desenvolve uma discussão bastante ampla sobre essa distinção.

${ }^{9}$ Church (1956) a denomina "Cálculo singularmente funcional de primeira ordem".
} 
Esse constituirá a segunda metade - a metade proposicional - do algoritmo. Uma primeira metade, constando de dois passos específicos à Lógica de Predicados Monádicos de Primeira Ordem, será acrescentada. $\mathrm{O}$ método aqui desenvolvido, a exemplo do método desenvolvido por Sautter (2013) utiliza resultados e técnicas bem conhecidos na literatura $^{10}$; sua originalidade e interesse residem na possibilidade da discussão in concreto do problema de demarcação entre o gráfico e o não-gráfico e, também, a possibilidade da discussão de problemas fundacionais, tal como o problema da caracterização das noções lógicas em termos de informação (e não em termos de verdade). O método, tanto em sua aplicação à Lógica Proposicional Clássica, como em sua aplicação à Lógica de Predicados Monádicos de Primeira Ordem, é bastante ineficaz. O método aqui desenvolvido também é interessante por revelar as relações entre a Lógica de Predicados (Monádicos) de Primeira Ordem e a silogística em sua forma expandida, tal como essa foi desenvolvida ao longo do século XIX.

Na próxima seção apresentarei alguns antecedentes históricos do desenvolvimento de estudos sobre a Lógica de Predicados Monádicos de Primeira Ordem, bem como resultados notáveis acerca dela, que serão empregados no método aqui proposto. A seguir, apresentarei o método e o exemplificarei. Finalmente, discutirei brevemente a possibilidade de estender o resultado aqui obtido a outras lógicas.

\section{ANTECEDENTES HistóRicos E RESUltados NotÁveis}

Recorrerei a resultados conhecidos para uma linguagem de

\footnotetext{
${ }^{10}$ Basicamente, eles dependem da existência de formas normais conjuntivas completas.
} 
primeira ordem L sem igualdade e cujos únicos símbolos não-lógicos são símbolos de predicados unários (CELLUCCI 1999, p.171). Além disso, esses resultados não dizem respeito a um método de prova geral para a Lógica de Predicados Monádicos de Primeira Ordem, mas apenas ao problema da validade, ou seja, à determinação das sentenças que são verdades lógicas.

Há, basicamente, dois métodos de prova disponíveis na literatura: um método deriva de trabalhos de Bernays e Schönfinkel (CHURCH 1956, p. 253), e o outro deriva de trabalhos de Behmann e Quine (CHURCH 1956, p.293294).

O método de Bernays e Schönfinkel, que não será utilizado neste trabalho, segue-se do seguinte resultado sobre cardinalidade de domínios de indivíduos:

Teorema 1 (Teorema de Bernays e Schönfinkel). Se uma fórmula bem formada do Cálculo Singularmente Funcional de Primeira Ordem é válida em um domínio de $2^{\mathrm{n}}$ indivíduos, em que $n$ é o número de diferentes variáveis funcionais [da fórmula], então ela é válida em todos os domínios de indivíduos (CHURCH 1956, p. 253).

Utilizarei o método de Behmann e Quine. Esse método depende da existência de uma forma normal de Herbrand para toda sentença da Lógica de Predicados Monádicos de Primeira Ordem (CHURCH 1956, p.257-258). A definição da forma normal de Herbrand utiliza a seguinte definição auxiliar: 
Definição 2 (Quantificação elementar). Por uma quantificação elementar entende-se uma fórmula de $\mathrm{L}$ da forma $\exists x$ $\Lambda_{\mathrm{i}=1}^{\mathrm{n}} \varphi_{\mathrm{i}}$, em que cada $\varphi_{\mathrm{i}}$ é ou $\mathrm{Px}$ ou $\neg \mathrm{P} x$, para algum símbolo de predicado monádico P (CELLUCCI 1999, p. 171).

Uma sentença em forma normal de Herbrand é caracterizada do seguinte modo:

Definição 3 (Forma Normal de Herbrand). Uma sentença de L está em forma normal de Herbrand se, e somente se, ela é uma combinação verofuncional de quantificações elementares.

A forma normal de Herbrand estabelece uma conexão precisa entre as sentenças da Lógica de Predicados Monádicos de Primeira Ordem e a Silogística Ampliada do Século $\mathrm{XIX}^{11}$. As quantificações elementares podem ser representadas na Silogística Ampliada como, por exemplo, proposições da forma "Algum $\Sigma$ é $\Pi$ ", em que $\Sigma$ e $\Pi$ são conjunções de termos afirmativos e negativos que estão por literais de fórmulas atômicas da Lógica de Predicados Monádicos de Primeira Ordem. Por exemplo, $\exists x(\mathrm{P} x \wedge \neg \mathrm{Q} x \wedge \neg \mathrm{R} x \wedge \mathrm{S} x)$ pode ser representado, na Silogística Ampliada, por "Algum $\mathrm{P}$ é QRS", ou por "Algum QS é PR"12, e assim por diante. Nesse sentido, as sentenças da Lógica de Predicados Monádicos de Primeira Ordem são combinações verofuncionais de juízos categóricos com termos complexos.

\footnotetext{
${ }^{11}$ Essa Silogística Ampliada pode ser caracterizada pelo emprego de termos negativos e pelo emprego de termos complexos. Por exemplo, o manual de lógica mais popular do século XIX - "Studies and Exercises in Formal Logic" (1884), de John Neville Keynes - utiliza amplamente termos negativos, a ponto de desenvolver, a partir deles, um octógono de oposições e um método diagramático similar ao método de Euler; e, por exemplo, o manual de lógica de Lewis Carroll "The Game of Logic" (1887) - utiliza termos negativos e termos complexos, esses na forma de conjunção de termos.

${ }^{12}$ Uma letra sublinhada indica um termo negativo.
} 
Pode-se demonstrar o seguinte resultado:

Teorema 2 (Teorema da Forma Normal de Herbrand). Para toda sentença $\varphi$ de L, a forma normal de Herbrand $\psi$ de $\varphi$ é tal que $k_{\mathrm{L}} \varphi \leftrightarrow \psi($ CELLUCCI 1999, p. 172).

O método aqui proposto resulta do seguinte teorema:

Teorema 3 (Teorema Fundamental). Seja $\Delta U\{\varphi\}$ um conjunto finito de sentenças de L, $\varphi^{\mathrm{H}}$ a forma normal de Herbrand de $\varphi$, e $\Gamma^{\mathrm{H}}$ o conjunto das formas normais de Herbrand das sentenças de $\Gamma$. Tem-se que $\Delta F_{L} \varphi$ se, e somente se, $\Delta^{\mathrm{H}} k_{\mathrm{L}} \varphi^{\mathrm{H}}$.

Demonstração. Suponha que $\Delta=\left\{\psi_{1}, \ldots, \psi_{n}\right\}$ e que $\Delta F_{L} \varphi$. Por $n$ aplicações do Teorema (Restrito) da Dedução, $\Delta F_{L} \varphi$ se, e somente se, $k_{\mathrm{L}} \psi_{1} \rightarrow\left(\ldots\left(\psi_{n} \rightarrow \varphi\right) \ldots\right)$. Pelo Teorema da Forma Normal de Herbrand, $k_{\mathrm{L}} \psi_{1} \rightarrow\left(\ldots\left(\psi_{n} \rightarrow \varphi\right) \ldots\right)$ se, e somente se, $k_{\mathrm{L}}\left[\psi_{1} \rightarrow\left(\ldots\left(\psi_{\mathrm{n}} \rightarrow \varphi\right) \ldots\right)\right]^{\mathrm{H}}$. Pela definição da forma normal de Herbrand, $k_{\mathrm{L}}\left[\psi_{1} \rightarrow\left(\ldots\left(\psi_{n} \rightarrow \varphi\right)\right.\right.$ $\ldots)]^{\mathrm{H}}$ se, e somente se, $k_{\mathrm{L}} \psi_{1}{ }^{\mathrm{H}} \rightarrow\left(\ldots\left(\psi_{n}{ }^{\mathrm{H}} \rightarrow \varphi^{\mathrm{H}}\right) \ldots\right)$. Por $n$ aplicações do Teorema (Restrito) da Dedução, $k_{\mathrm{L}} \psi_{1}{ }^{\mathrm{H}} \rightarrow($ $\left.\ldots\left(\psi_{n}{ }^{\mathrm{H}} \rightarrow \varphi^{\mathrm{H}}\right) \ldots\right)$ se, e somente se, $\left.\left\{\psi_{1}{ }^{\mathrm{H}}, \ldots, \psi_{n}\right\}\right|_{L} \varphi^{\mathrm{H}}$, ou seja, $\Delta^{\mathrm{H}} F_{\mathrm{L}} \varphi^{\mathrm{H}}$.

Pelo Teorema da Compacidade, $\Gamma k_{\mathrm{L}} \varphi$ se, e somente se, existe um conjunto finito $\Delta \subseteq \Gamma$ tal que $\Delta k_{\mathrm{L}} \varphi$.

\section{O MÉTOdo de Prova}

O método de prova, descrito na figura abaixo, consiste de duas etapas. A etapa inicial, propriamente quantificacional, consiste em: 
1. Colocar as premissas e conclusão em forma normal de Herbrand (FNH).

2. Completar essas formas normais de Herbrand relativamente aos predicados monádicos que ocorrem no argumento, quer dizer, cada quantificação elementar será uma conjunção de fórmulas atômicas da Lógica de Predicados Monádicos de Primeira Ordem, de tal modo que todos os predicados monádicos ocorram em todas as fórmulas atômicas. Esse "completamento" é necessário, porque permitirá a correta comparação de distintas quantificações elementares no processo de passagem da Lógica de Predicados Monádicos de Primeira Ordem para a Lógica Proposicional. O resultado é que premissas e conclusão se encontrarão em forma normal de Herbrand completa (FNHC) relativamente aos predicados monádicos do argumento.

3. Realizar a transição para a Lógica Proposicional, substituindo mesmas quantificações elementares por mesmas proposições atômicas, e distintas quantificações elementares por distintas proposições atômicas.

A etapa proposicional do método consiste, à parte o teste preliminar para eliminar caso limite em que a conclusão é tautológica, em colocar premissas e conclusão em forma normal conjuntiva completa (FNCC) relativamente às proposições atômicas do argumento ${ }^{13}$. O último passo consiste em estabelecer se cada conjuntivo da FNCC da conclusão também é conjuntivo da FNCC de alguma pre-

\footnotetext{
${ }^{13}$ Essa exigência de "completamento" é, novamente, estabelecida para permitir a correta comparação de premissas e conclusão.
} 
missa (argumento válido) ou não (argumento inválido). O processo é bastante ineficaz e o último passo, propriamente diagramático, perde rapidamente o seu caráter sinóptico ao se examinarem argumento mais complexos.

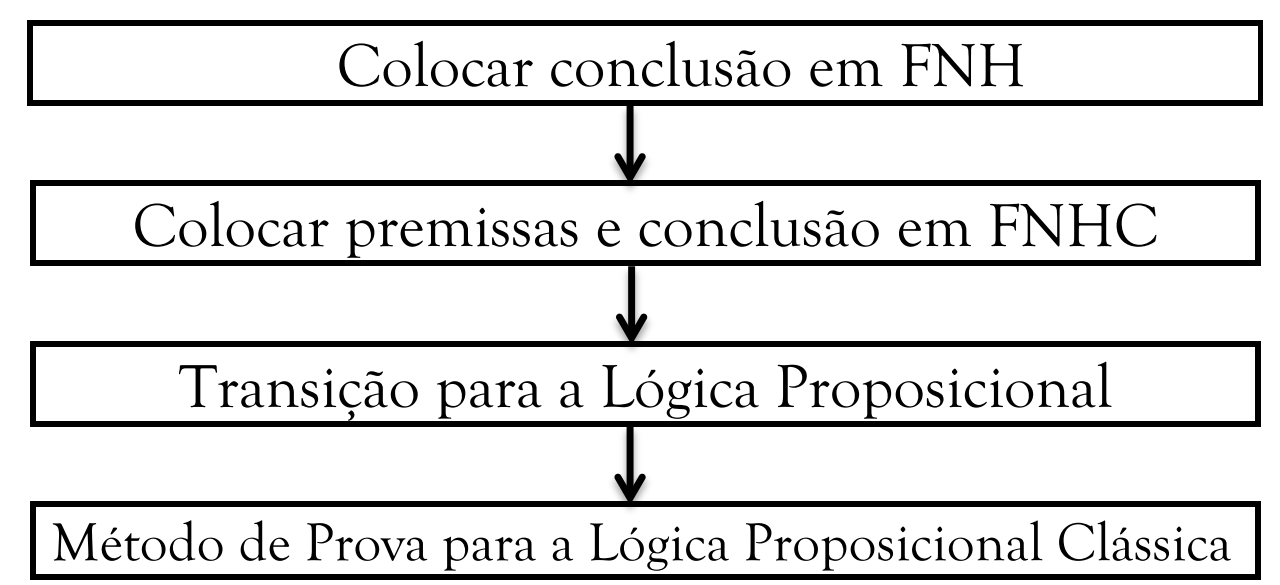

Figura 3: Método de prova para Lógica de Predicados Monádicos de Primeira Ordem: "FNH" é a forma normal de Herbrand, e "FNHC" é a forma normal de Herbrand completa relativa aos predicados monádicos do argumento.

$\mathrm{Na}$ exemplificação do método apresentarei sua aplicação completa a um modo silogístico válido e sua aplicação completa a um modo inválido.

Primeiro mostrarei que o modo EIO da $1^{\text {a }}$ Figura (FERIO) é válido.

$O$ esquema argumentativo FERIO é expresso, em linguagem natural, do seguinte modo: Nenhum $M$ é $P$ (premissa maior). Algum $S$ é $M$ (premissa menor). Portanto, algum $S$ não é $P$ (conclusão).

FERIO é expresso, na linguagem da Lógica de Predicados, por:

$\forall x(\mathrm{M} x \rightarrow \mathrm{P} x)$ (premissa maior)

$\exists x(\mathrm{~S} x \wedge \mathrm{M} x)$ (premissa menor) 
$\therefore \exists x(\mathrm{~S} x \wedge \neg \mathrm{Px})($ conclusão)

Após o primeiro passo - a obtenção da forma normal de Herbrand das premissas e conclusão - resulta:

$\neg \exists x(\mathrm{M} x \wedge \mathrm{Px})$ (premissa maior)

$\exists x(\mathrm{~S} x \wedge \mathrm{Mx})$ (premissa menor)

$\therefore \exists x(\mathrm{~S} x \wedge \neg \mathrm{Px})($ conclusão $)$

Após o segundo passo - a obtenção da forma normal completa de Herbrand das premissas e conclusão - resulta:

$\neg \exists x(\mathrm{~S} x \wedge \mathrm{M} x \wedge \mathrm{P} x) \wedge \neg \exists x(\neg \mathrm{S} x \wedge \mathrm{M} x \wedge \mathrm{P} x)$ (premissa maior)

$\exists x(\mathrm{~S} x \wedge \mathrm{M} x \wedge \mathrm{Px}) \vee \exists x(\mathrm{~S} x \wedge \mathrm{M} x \wedge \neg \mathrm{P} x)$ (premissa menor)

$\therefore \exists x(\mathrm{~S} x \wedge \mathrm{M} x \wedge \neg \mathrm{P} x) \vee \exists x(\mathrm{~S} x \wedge \neg \mathrm{Mx} \wedge \neg \mathrm{Px})$ (conclusão)

Após o terceiro passo - transição para a Lógica Proposicional - resulta:

$\neg p \wedge \neg q$ (premissa maior)

$\mathrm{p} \vee \mathrm{r}$ (premissa menor)

$\therefore \mathrm{r} \vee \mathrm{s}$ (conclusão)

A forma normal disjuntiva da conclusão $-r \vee s-$ não é uma tautologia, por isso obtenhamos a forma normal conjuntiva das premissas e conclusão. Elas, contudo, já se encontram em forma normal conjuntiva. 
O passo seguinte e final é a obtenção da forma normal conjuntiva completa das premissas e conclusão:

Premissa maior:

$(\neg p \vee q \vee r \vee s) \wedge(\neg p \vee q \vee r \vee \neg s) \wedge(\neg p \vee q \vee \neg r \vee s) \wedge(\neg p \vee$ $\neg \mathrm{q} \vee \mathrm{r} \vee \mathrm{s}) \wedge(\neg \mathrm{p} \vee \mathrm{q} \vee \neg \mathrm{r} \vee \neg \mathrm{s}) \wedge(\neg \mathrm{p} \vee \neg \mathrm{q} \vee \mathrm{r} \neg \mathrm{s}) \wedge(\neg \mathrm{p} \vee \neg \mathrm{q} \vee$ $\neg \mathrm{r} \vee \mathrm{s}) \wedge(\neg \mathrm{p} \vee \neg \mathrm{q} \vee \neg \mathrm{r} \vee \neg \mathrm{s}) \wedge(\mathrm{p} \vee \neg \mathrm{q} \vee \mathrm{r} \vee \mathrm{s}) \wedge(\mathrm{p} \vee \neg \mathrm{q} \vee \mathrm{r} \vee$ $\neg \mathrm{s}) \wedge(\mathrm{p} \vee \neg \mathrm{q} \vee \neg \mathrm{r} \vee \mathrm{s}) \wedge(\mathrm{p} \vee \neg \mathrm{q} \vee \neg \mathrm{r} \vee \neg \mathrm{s})$

Premissa menor:

$(\mathrm{p} \vee \mathrm{q} \vee \mathrm{r} \vee \mathrm{s}) \wedge(\mathrm{p} \vee \mathrm{q} \vee \mathrm{r} \vee \neg \mathrm{s}) \wedge(\mathrm{p} \vee \neg \mathrm{q} \vee \mathrm{r} \vee \mathrm{s}) \wedge(\mathrm{p} \vee \neg \mathrm{q} \vee$ $r \vee \neg s)$

Conclusão:

$(\mathrm{p} \vee \mathrm{q} \vee \mathrm{r} \vee \mathrm{s}) \wedge(\neg \mathrm{p} \vee \mathrm{q} \vee \mathrm{r} \vee \mathrm{s}) \wedge(\mathrm{p} \vee \neg \mathrm{q} \vee \mathrm{r} \vee \mathrm{s}) \wedge(\neg \mathrm{p} \vee \neg \mathrm{q}$ $\vee \mathrm{r} \vee \mathrm{s}$ )

O modo é válido porque o primeiro conjuntivo da conclusão está disponível (somente) na premissa maior, o segundo conjuntivo está disponível (somente) na premissa menor, o terceiro conjuntivo está disponível em ambas as premissas, e o quarto conjuntivo está disponível (somente) na premissa maior. Além disso, ambas premissas são indispensáveis para a dedução da conclusão.

Agora mostrarei que o modo AEE da 1ª Figura é inválido.

O esquema argumentativo AEE da $1^{\text {a }}$ Figura é expresso, na linguagem natural, do seguinte modo: Todo $M$ é $P$ (premissa maior). Nenhum S é $M$ (premissa menor). Portanto, nenhum $S$ é $P$ (conclusão).

$\mathrm{O}$ esquema argumentativo AEE da $1^{\text {a }}$ Figura é expresso, na Linguagem da Lógica de Predicados, por: 
$\forall x(\mathrm{M} x \rightarrow \mathrm{P} x)$ (premissa maior)

$\forall x(\mathrm{~S} x \rightarrow \neg \mathrm{M} x)$ (premissa menor)

$\therefore \forall x(\mathrm{~S} x \rightarrow \neg \mathrm{P} x)($ conclusão)

Após o primeiro passo - a obtenção da forma normal de Herbrand das premissas e conclusão - resulta:

$\neg \exists x(\mathrm{M} x \wedge \neg \mathrm{P} x)$ (premissa maior)

$\neg \exists x(\mathrm{~S} x \wedge \mathrm{M} x)$ (premissa menor)

$\therefore \neg \exists x(S x \wedge P x)($ conclusão)

Após o segundo passo - a obtenção da forma normal completa de Herbrand das premissas e conclusão - resulta:

$\neg \exists x(\mathrm{~S} x \wedge \mathrm{M} x \wedge \neg \mathrm{Px}) \wedge \neg \exists x(\neg \mathrm{S} x \wedge \mathrm{M} x \wedge \neg \mathrm{P} x)$ (premissa maior)

$\neg \exists x(\mathrm{~S} x \wedge \mathrm{M} x \wedge \mathrm{P} x) \wedge \neg \exists x(\mathrm{~S} x \wedge \mathrm{M} x \wedge \neg \mathrm{P} x)$ (premissa menor)

$\therefore \neg \exists x(\mathrm{~S} x \wedge \mathrm{M} x \wedge \mathrm{Px}) \wedge \neg \exists x(\mathrm{~S} x \wedge \neg \mathrm{Mx} \wedge \mathrm{Px})($ conclusão)

Após o terceiro passo - transição para a Lógica Proposicional - resulta:

$\mathrm{p} \wedge \mathrm{q}$ (premissa maior)

$\mathrm{r} \wedge \mathrm{p}$ (premissa menor)

$\therefore \mathrm{r} \wedge \mathrm{s}($ conclusão $)$

A forma normal disjuntiva da conclusão $-\mathrm{r} \wedge \mathrm{s}-$ não PHILÓSOPHOS, GOIÂNIA, V.20, N.1, P.145-162, JAN./JUN. 2015. 
é uma tautologia, por isso obtenhamos a forma normal conjuntiva das premissas e conclusão. Elas, contudo, já se encontram em forma normal conjuntiva.

O passo seguinte e final é a obtenção da forma normal conjuntiva completa das premissas e conclusão:

Premissa maior:

$(\mathrm{p} \vee \mathrm{q} \vee \mathrm{r} \vee \mathrm{s}) \wedge(\mathrm{p} \vee \mathrm{q} \vee \mathrm{r} \vee \neg \mathrm{s}) \wedge(\mathrm{p} \vee \mathrm{q} \vee \neg \mathrm{r} \vee \mathrm{s}) \wedge(\mathrm{p} \vee \neg \mathrm{q} \vee$ $\mathrm{r} \vee \mathrm{s}) \wedge(\mathrm{p} \vee \mathrm{q} \vee \neg \mathrm{r} \vee \neg \mathrm{s}) \wedge(\mathrm{p} \vee \neg \mathrm{q} \vee \mathrm{r} \neg \mathrm{s}) \wedge(\mathrm{p} \vee \neg \mathrm{q} \vee \neg \mathrm{r} \vee \mathrm{s}) \wedge$ $(\mathrm{p} \vee \neg \mathrm{q} \vee \neg \mathrm{r} \vee \neg \mathrm{s}) \wedge(\neg \mathrm{p} \vee \mathrm{q} \vee \mathrm{r} \vee \mathrm{s}) \wedge(\neg p \vee \mathrm{q} \vee \mathrm{r} \vee \neg \mathrm{s}) \wedge(\neg p \vee$ $\mathrm{q} \vee \neg \mathrm{r} \vee \mathrm{s}) \wedge(\neg \mathrm{p} \vee \mathrm{q} \vee \neg \mathrm{r} \vee \neg \mathrm{s})$

Premissa menor:

$(\mathrm{p} \vee \mathrm{q} \vee \mathrm{r} \vee \mathrm{s}) \wedge(\mathrm{p} \vee \mathrm{q} \vee \mathrm{r} \vee \neg \mathrm{s}) \wedge(\mathrm{p} \vee \neg \mathrm{q} \vee \mathrm{r} \vee \mathrm{s}) \wedge(\neg \mathrm{p} \vee \mathrm{q} \vee$ $\mathrm{r} \vee \mathrm{s}) \wedge(\mathrm{p} \vee \neg \mathrm{q} \vee \mathrm{r} \vee \neg \mathrm{s}) \wedge(\neg \mathrm{p} \vee \mathrm{q} \vee \mathrm{r} \neg \mathrm{s}) \wedge(\neg \mathrm{p} \vee \neg \mathrm{q} \vee \mathrm{r} \vee \mathrm{s}) \wedge$ $(\neg p \vee \neg q \vee r \vee \neg s) \wedge(p \vee q \vee \neg r \vee s) \wedge(p \vee q \vee \neg r \vee \neg s) \wedge(p \vee$ $\neg q \vee \neg r \vee \neg s) \wedge(p \vee \neg q \vee \neg r \vee \neg s)$

Conclusão:

$(\mathrm{p} \vee \mathrm{q} \vee \mathrm{r} \vee \mathrm{s}) \wedge(\mathrm{p} \vee \mathrm{q} \vee \mathrm{r} \vee \neg \mathrm{s}) \wedge(\mathrm{p} \vee \neg \mathrm{q} \vee \mathrm{r} \vee \mathrm{s}) \wedge(\neg \mathrm{p} \vee \mathrm{q} \vee$ $\mathrm{r} \vee \mathrm{s}) \wedge(\mathrm{p} \vee \neg \mathrm{q} \vee \mathrm{r} \vee \neg \mathrm{s}) \wedge(\mathrm{p} \vee \mathrm{q} \vee \mathrm{r} \neg \mathrm{s}) \wedge(\neg p \vee \neg \mathrm{q} \vee \mathrm{r} \vee \mathrm{s}) \wedge$ $(\neg p \vee \neg q \vee r \vee \neg s) \wedge(p \vee q \vee \neg r \vee s) \wedge(p \vee \neg q \vee \neg r \vee \neg s) \wedge(\neg p$ $\vee q \vee \neg r \vee s) \wedge(\neg p \vee \neg q \vee \neg r \vee s)$

O modo é inválido porque o último conjuntivo da conclusão $-\neg p \vee \neg q \vee \neg r \vee s-$ não está disponível nas premissas.

Complementarei, a seguir, a exemplificação do método com exercícios propostos por Lewis Carroll (1887), relacionados à silogística ampliada.

Carroll (1887, p.86) fornece o seguinte par de premissas e pede uma conclusão silogística, caso exista: 
Premissa 1: Ninguém infeliz gargalha. ${ }^{14}$

Premissa 2: Ninguém feliz se lamenta. ${ }^{15}$

Utilizarei, na formalização, o seguinte dicionário:

Fx: x é feliz.

Gx: x gargalha.

Lx: $\mathrm{x}$ se lamenta.

O par de premissas é expresso, na linguagem da Lógica de Predicados e com o auxílio do dicionário, por:

Premissa 1: $\forall \mathrm{x}(\neg \mathrm{Fx} \rightarrow \neg \mathrm{Gx})$

Premissa 2: $: \forall x(F x \rightarrow \neg L x)$

Após a obtenção da forma normal de Herbrand resulta:

Premissa 1: $\neg \mathrm{x}(\neg \mathrm{Fx} \wedge \mathrm{Gx})$

Premissa 2: $\neg \exists x(F x \wedge L x)$

Após a transição para a Lógica Proposicional resulta:

Premissa 1: $\mathrm{p} \wedge \mathrm{q}$

Premissa 2: $\mathrm{r} \wedge \mathrm{s}$

Há uma multiplicidade de conclusões, mas o que é solicitado é uma conclusão silogística, ou seja, uma conclusão em $\mathrm{G}$ e em L, uma vez que $\mathrm{F}$ é o termo médio.

Segue-se que a conclusão almejada é $\mathrm{p} \wedge \mathrm{r}$, ou seja,

\footnotetext{
${ }^{14}$ No unhappy people chuckle.

${ }^{15}$ No happy people groan.
} 
$\neg \exists x(\neg F x \wedge \mathrm{Gx} \wedge \mathrm{Lx}) \wedge \neg \exists x(\mathrm{Fx} \wedge \mathrm{Gx} \wedge \mathrm{Lx})$, ou seja, $\neg \exists x(G x \wedge L x)$, ou seja, ninguém que gargalha se lamenta.

Carroll (1887, p.87) fornece o seguinte par de premissas e, novamente, pede uma conclusão silogística, caso exista:

Premissa 1: Nenhum judeu idoso é um moleiro obeso. ${ }^{16}$

Premissa 2: Todos os meus amigos são moleiros obesos. ${ }^{17}$

Utilizarei, na formalização, o seguinte dicionário:

Jx: x é judeu; Ix: x é idoso; Mx: X é moleiro; Ox: x é obeso; Ax: $\mathrm{x}$ é meu amigo.

O par de premissas é expresso, na linguagem da Lógica de Predicados e com auxílio do dicionário, por:

Premissa 1: $\forall \mathrm{x}[(\mathrm{Jx} \wedge \mathrm{Ix}) \rightarrow \neg(\mathrm{Mx} \wedge \mathrm{Ox})]$

Premissa 2: $\forall \mathrm{x}[\mathrm{Ax} \wedge(\mathrm{Mx} \wedge \mathrm{Ix})]^{18}$

Após a obtenção da forma normal de Herbrand e rearranjo de conjuntivos (visando o destaque do termo médio) resulta:

Premissa 1: $\neg \exists x[(J x \wedge O x) \wedge(\mathrm{Mx} \wedge \mathrm{Ix})]$

Premissa 2: $\neg \exists x[(\mathrm{Ax} \wedge \neg(\mathrm{Mx} \wedge \mathrm{Ix})]$

Façamos $\mathrm{Kx}{ }_{\text {def. }} \mathrm{Mx} \wedge \mathrm{Ix}$ e $\mathrm{Lx}=_{\text {def }} \mathrm{Jx} \wedge \mathrm{Ox}$. Após a ob-

\footnotetext{
${ }^{16}$ No old Jews are far millers.

${ }^{17}$ All my friends are old millers.

${ }^{18}$ Para Lewis Carroll as proposições categóricas universais afirmativas são proposições duplas, ou seja, elas afirmam a existência de seus sujeitos. Aqui fornecerei um tratamento tradicional às universais afirmativas.
} 
tenção da forma normal completa de Herbrand resulta:

Premissa 1: $\neg \exists x(A x \wedge \operatorname{Lx} \wedge \neg \mathrm{Kx}) \wedge \neg \exists x(\neg A x \wedge \operatorname{Lx} \wedge$ $\neg \mathrm{Kx})$

Premissa 2: $\neg \exists x(A x \wedge L x \wedge \mathrm{Kx}) \wedge \neg \exists x(A x \wedge \neg L x \wedge \mathrm{Kx})$

Após a transição para a Lógica Proposicional resulta:

Premissa 1: $\mathrm{p} \wedge \mathrm{q}$

Premissa 2: $\mathrm{r} \wedge \mathrm{s}$

Há, novamente, uma multiplicidade de conclusões, mas o que é solicitado é uma conclusão silogística, ou seja, uma conclusão em A e em L, uma vez que K é o termo médio.

Segue-se que a conclusão almejada é p $\wedge \mathrm{r}$, ou seja $\neg \exists x$ $(\mathrm{Ax} \wedge \mathrm{Lx} \wedge \neg \mathrm{Kx}) \wedge \neg \exists \mathrm{x}(\mathrm{Ax} \wedge \mathrm{Lx} \wedge \mathrm{Kx})$, ou seja, $\neg \exists \mathrm{x}$ $(A x \wedge \mathrm{Lx})$, ou seja, nenhum judeu obeso é meu amigo.

\section{CONSIDERAÇÕES FINAIS}

Obtive um método de prova para a Lógica de Predicados Monádicos de Primeira Ordem que é dividido em duas partes: uma parte propriamente quantificacional e a outra parte proposicional. Cada parte requereu a utilização de uma forma normal completa. $O$ último passo do método é diagramático de um tipo muito especial: exige-se a mera constatação da disposição de certas representações.

O método pode ser, aparentemente, estendido a outras lógicas, desde que essas tenham uma forma normal completa. No entanto, duas restrições necessitam ser esclarecidas: 
1. A forma normal precisa ser tal que o resultado é uma combinação verofuncional de "átomos", de tal modo a permitir a substituição desses “átomos" por proposições atômicas da Lógica Proposicional Clássica e, desse modo, ingressar na parte proposicional do método. Por exemplo, as quantificações elementares cumprem esse papel no caso da Lógica de Predicados Monádicos de Primeira Ordem.

2. A mera existência de uma forma normal com as características exigidas na restrição anterior não garante que a existência de uma forma normal (com as características exigidas na restrição anterior) completável. Por exemplo, pode muito bem ser o caso que uma lógica modal tenha uma forma normal com as características exigidas na restrição anterior mas essa forma normal não seja completável, porque há um número infinito de modalidades distintas.

método é bastante ineficaz: ele exige muito trabalho preliminar antes que se possam aplicar meras operações de inspeção de representações gráficas. Além disso, ele sugere que, mesmo que admitíssemos a noção de informação como fundacionalmente mais adequada do que a noção de verdade, esta última poderia operar como um elemento ideal, simplificando enormemente o trabalho de obtenção de provas.

Abstract: I provide a proof method for First Order Monadic Predicate Logic. This method uses the Normal Form of Herbrand and the Disjunctive and Conjunctive Normal Forms for Propositional Logic. The validity is determined by mere inspection of the presence and arrangement of formulas that act as informational atoms. The exact relationship between First Order Monadic Predicate Logic and the extended syllogistic developed during the nineteenth century is established by the Normal Form of Herbrand.

Keywords: Symbolic knowledge; Monadic Predicate Logic; Extended syllogistic; Herbrand. 


\section{REFERÊNCIAS}

CARROLL, Lewis. The Game of Logic. London and New York: Macmillan, 1887.

CELLUCCI, Carlo. The decidability of Syllogism. In: EGIDI, Rosaria (ed.) In Search of a New Humanism. The philosophy of Georg Henrik von Wright. Dordrecht: Kluwer, 1999. P. 171-174.

CHURCH, Alonzo. Introduction to Mathematical Logic. Volume I. Priceton: Princeton University Press, 1956.

ESQUISABEL, Oscar Miguel. Representing and Abstracting: An analysis of Leibniz's concept of symbolic knowledge. In: LASSALLE CASANAVE, Abel (org.) Symbolic Knowledge from Leibniz to Husserl. London: College Publications, 2012. p. 1-49.

KEYNES, John Neville. Studies and Exercises in Formal Logic. London: Macmillan, 1884.

MENDONÇA, Bruno Ramos. Conhecimento Simbólico em John Venn. Dissertação (mestrado) - Universidade Federal de Santa Maria, Centro de Ciências Sociais e Humanas, Programa de Pós-Graduação em Filosofia, 2013.

SAUTTER, Frank Thomas. Linear K. In: LASSALLE CASANAVE, Abel \& SAUTTER, Frank Thomas (orgs.) Visualização nas Ciências Formais. London: College Publications, 2012. p. 145-161.

SAUTTER, Frank Thomas. Leitmotiv de Hilbert y Ackermann. In: ESQUISABEL, Oscar Miguel \& SAUTTER, Frank Thomas (orgs.) Conocimiento Simbólico y Conocimi- 
ento Gráfico. Ciudad de Buenos Aires: Academia Nacional de Ciencias de Buenos Aires, 2013. p. 71-79.

SECCO, Gisele Dalva. Entre Provas e Experimentos. Uma leitura wittgensteiniana das controvérsias em torno do Teorema das Quatro Cores. Tese (doutorado) - Pontifícia Universidade Católica do Rio de Janeiro, Departamento de Filosofia, 2013.

SHIMOJIMA, Atsushi. The Graphic-Linguistic Distinction: Exploring Alternatives. Artificial Intelligence Review., v.15, 2001. p. 5-27. 